\title{
Point-of-Care Testing for D-Dimer in the Diagnosis of Venous Thromboembolism in Primary Care: A Narrative Review
}

\author{
Christopher P. Price (D) - Matthew Fay · Rogier M. Hopstaken
}

Received: October 23, 2020 / Published online: December 2, 2020

(C) The Author(s) 2020

\begin{abstract}
Venous thromboembolism (VTE) is regarded as a significant cause of mortality and disability, affecting 1-2 per 1000 people annually, presenting with a relatively wide range of symptoms, which can pose a diagnostic challenge. Historically, people in whom VTE is suspected will have been taken to hospital for diagnosis and treatment; however, a high proportion of patients are found not to have VTE. Concerns have been expressed about potential delays in treatment, with the risk of additional morbidity and disability, and death. Diagnostic strategies are typically based on the use of a clinical prediction rule to determine the pre-test probability, complemented with a measurement of D-dimer, with confirmation by imaging assessment. This narrative review explores the literature on the use of point-of-care testing (POCT) for the measurement of D-dimer, as part of a clinical decision rule, for the diagnosis of deep
\end{abstract}

C. P. Price $(\square)$

Nuffield Department of Primary Care Health Sciences, University of Oxford, Oxford, UK e-mail: cpprice1@gmail.com

M. Fay

Affinity Care, The Willows Medical Practice, Queensbury, UK

R. M. Hopstaken

Star-Shl Diagnostic Centres, Etten-Leur, The Netherlands vein thrombosis (DVT) and pulmonary embolism (PE) in the primary care setting. In the two main prospective management (validation) studies that included D-dimer POCT or similar technologies, with a total cohort of 1600 participants, DVT was ruled out in $49 \%$ of patients, with a false negative rate of $1.4 \%$, whereas PE was ruled out in $45 \%$ of patients, with a false negative rate of $1.5 \%$. This suggests that uptake of POCT D-dimer in primary care has the potential to reduce the number of referrals to hospitals for imaging confirmatory investigation, with consequent cost savings. Thus, adopting POCT for D-dimer in primary care can offer clinical and cost benefits, particularly when quantitative POCT assays are being used. Furthermore, POCT should be undertaken in collaboration with the local laboratories to ensure the harmonisation of D-dimer methods and quality assurance to improve the diagnosis of VTE.

Keywords: D-dimer; Deep vein thrombosis; Diagnostic performance; Point-of-care testing; Primary care; Pulmonary embolism; Venous thromboembolism

Key Summary Points

Why carry out this study? 
There is concern about delays in diagnosis and treatment of venous thromboembolism (VTE) due to its varied presentation in primary care.

This review sought to assess whether the use of point-of-care testing (POCT) for the measurement of D-dimer, as part of a clinical decision rule, in patients presenting in primary care with suspected VTE, improves health outcomes.

What was learned from the study?

POCT for D-dimer with a clinical decision rule can rule out a diagnosis of VTE in the primary care setting, thereby reducing the need for referral to hospital in patients without the condition.

Age-related cut-off values for the D-dimer test can be helpful in the elderly, and POCT D-dimer testing in primary care should be supported by a local laboratory.

Evidence investigating the use of POCT for D-dimer specifically in primary care is limited, although study patient cohorts are relatively large.

\section{DIGITAL FEATURES}

This article is published with digital features to facilitate understanding of the article. You can access the digital features on the article's associated Figshare page. To view digital features for this article go to https://doi.org/10.6084/m9. figshare.13251902

\section{INTRODUCTION}

Venous thromboembolism (VTE) comprises deep vein thrombosis (DVT) and pulmonary embolism (PE), with an estimated average incidence rate ranging from 104 to 183 per 100,000 person-years [1]. The signs and symptoms of VTE at the time of presentation can be notably non-specific [2], with VTE diagnosed in only $10 \%$ of those in whom it is suspected [3]. Concern has been expressed about the delay in diagnosis, particularly where clinical presentations are often less explicit $[4,5]$. Initiation of treatment is advised urgently to prevent additional morbidity, disability and the risk of death [6]. Other concerns include extra patient burden when unnecessarily referred to the hospital for additional examinations, and increased healthcare costs incurred through overuse of healthcare resources [7].

The most commonly accepted diagnostic strategy for VTE is based on the use of a clinical decision rule to determine the pre-test probability, complemented with a measurement of D-dimer, (possibly) followed by a confirmatory imaging assessment (compression ultrasonography [CUS] for DVT, and computed tomography pulmonary angiography [CTPA] for PE) [8]. The practical utility of the clinical decision rule is the safe identification of patients with suspected VTE who are at low risk, and therefore unlikely to require urgent hospital referral for further investigation. Over the years, several clinical decision rules have been developed for use in primary care, and have been shown to give a comparable performance [8]. Oudega et al. developed a set of clinical decision rules in 2005 using a study of 1295 patients with suspected DVT in whom patients with suspected PE were excluded [9]. The performance of this simplified decision rule was compared with the more established Wells score [10] and subsequently validated in 525 patients by Toll et al. in 2006 [11]. These derivation and validation studies [9-11] used quantitative laboratorybased D-dimer tests, and revealed that the decision rule could not only rule out a diagnosis of DVT safely, but also reduce the number of unnecessary patient referrals to secondary care for further investigations. Recently, the use of an age-related cut-off value for D-dimer has been proposed, offering an improved diagnostic performance of the decision rule, although this has not been widely adopted to date [3].

Most practice guidelines advocate the use of a clinical decision rule in combination with the measurement of D-dimer. Recent guidelines have supported the introduction of D-dimer measurement as part of a clinical decision rule when the method of detection has a D-dimer sensitivity of $\geq 95 \%[12,13]$. The use of agerelated D-dimer cut-off values (where diagnostic yield is increased in low- and medium-risk populations) [14], and the use of D-dimer where the risk score is below a fixed threshold [15], have also been recommended. Applying pointof-care testing (POCT) is suggested as an option 
if operated in collaboration with an accredited laboratory for reasons of quality control [16] and patient safety [15].

The National Institute for Health and Care Excellence (NICE) VTE guidelines, updated in March 2020, recommend the use of the Wells score for DVT and PE [17]. Review of the evidence indicated that if a patient is suspected with DVT, a two-level DVT Wells score should be offered to estimate the clinical probability of DVT $[17,18]$. Patients with a 'likely' DVT Wells score ( $\geq 2$ points) should be offered a proximal leg vein ultrasound scan within $4 \mathrm{~h}$ and confirmed with a D-dimer test if the scan results are negative. In cases where proximal leg vein ultrasound scan results cannot be obtained within $4 \mathrm{~h}$, a D-dimer test is recommended followed by a proximal leg vein ultrasound within $24 \mathrm{~h} \mathrm{[17].} \mathrm{In} \mathrm{patients} \mathrm{where} \mathrm{DVT} \mathrm{is} \mathrm{suspected}$ and when the two-level DVT Wells score is 'unlikely' ( $\leq 1$ point), a D-dimer test is recommended, with a proximal leg vein ultrasound performed if the D-dimer test result is positive [17].

Similarly, patients with suspected PE should be assessed using a two-level PE Wells score $[17,19]$. Those with a likely PE Wells score $(>4$ points) should be offered prompt CTPA or a suitable alternative [17]. D-dimer testing is recommended for patients with an 'unlikely' PE Wells score ( $\leq 4$ points), with imaging diagnosis methods undertaken if the test results are positive $[17,19]$.

The revised NICE guidelines support the use of a quantitative D-dimer test, with consideration given to the use of POCT if laboratory facilities are not immediately available $[17,20]$. Furthermore, when using point-of-care (POC) or laboratory D-dimer test, an age-adjusted D-dimer test threshold for people aged over 50 years should be considered $[17,20]$.

Analysis of five case studies by the All-Party Parliamentary Thrombosis Group demonstrated that the availability of diagnostic tools, such as the POC D-dimer tests, in primary care led to the redesigning of local community-based DVT pathways [21]. This not only improved primary care services and patient experience by allowing patients to be treated closer to their homes, but also facilitated substantial cost savings for the local health economy by reducing unnecessary hospital admissions [21]. This review aimed to summarise the diagnostic performance of POCT for D-dimer when used as an integral part of a clinical decision rule in adult patients presenting with symptoms of VTE in primary care, and to explore some of the practical implications of applying widespread POCT for D-dimer in primary care, including its cost-effectiveness.

\section{METHODS}

A search on PubMed, Cochrane Library, CINAHL and EMBASE databases was undertaken on November 25, 2019. The search was limited to English language publications over the period 2000-2019; this was considered the main period in which POCT technologies for D-dimer have evolved. Search terms included terms for D-dimer (fibrin fragment D) test, POCT, primary care (general practice), VTE, and their synonyms, including relevant medical subject headings; the same terms were used for each of the databases interrogated, with syntax adjusted according to the database.

A reviewer screened the citations using the title and abstract, and selected the studies for further investigation. Studies were included if they were conducted in a primary care setting; reported a randomised controlled, observational or validation study; and investigated the performance characteristics of POCT for D-dimer, used in combination with a clear decision rule with a reference standard for diagnosis, or the practical implications of applying POCT for D-dimer (e.g., cost-effectiveness). Case studies, study protocols and editorials were excluded, together with studies where other comorbidities were reported in the patient cohort. The references cited by identified systematic reviews were investigated for additional potentially relevant citations. This article is based on previously conducted studies and does not contain any new studies with human participants or animals performed by any of the authors. 


\section{RESULTS}

The database searches generated 32 unique citations, including two potentially relevant systematic reviews. Interrogation of the systematic review citation lists identified a further eight potentially relevant citations. Screening identified a total of eight articles relevant to the review aims.

\section{Evidence and Expected Outcomes}

Our search found two original (primary) studies investigating the use of POCT for D-dimer testing as part of a clinical decision rule, recruiting participants from primary care settings, one cohort of participants with suspected DVT [22] and the other with suspected PE [23]. A total of five secondary studies utilised the patient cohorts and data from the primary studies, identifying supplementary research questions (Table 1) [24-28]. An additional study investigated a secondary question related to the impact of using age-related cut-off values for D-dimer, recruiting a cohort of older patients residing in a nursing home (Table 1) [29].

\section{Clinical Effectiveness of POCT D-Dimer in DVT}

Buller et al., in 2009, evaluated the safety and effectiveness of using a clinical decision rule that included a qualitative POC D-dimer assay at initial presentation in primary care to exclude DVT [22]. They recruited 1028 consecutive patients with clinically suspected DVT, from approximately 300 practices in the Netherlands, into a prospective management study. Patients were managed based on the result of the Oudega clinical decision rule [9-11], which included the D-dimer assessment. The validated decision rule classified $49 \%$ of the study cohort to be at low risk of developing VTE, without the need for imaging tests and anticoagulation treatment. In the 3-month period following, 1.4\% (95\% confidence interval [95\% CI]: 0.6-2.9\%) of low-risk patients had VTE (Table 1) [22].
Geersing et al. used the same study population to compare the performance of a clinical decision rule with the clinical probability score for the presence of DVT as estimated by general practitioners (GPs; Table 1) [24]. The probability scores were defined as $<10 \%,<20 \%$ and $>$ $20 \%$, with $<10 \%$ and $<20 \%$ categorised as low risk. A confirmed diagnosis of DVT was established over the 90-day follow-up period by CUS, and/or venous thromboembolic complications, including death. The clinician probability score resulted in more patients being referred for imaging (79\% and $65 \%$ for $<10 \%$ and $<20 \%$ thresholds, respectively), compared with the decision rule (51\%). Both approaches were found to have a similar false negative rate (2.0\% [95\% CI $0.5-3.4 \%$ ] for the probability score). One limitation noted by the authors was that GPs may have been influenced by the use of the decision rule when calculating their probability score, resulting in the potential for incorporation bias.

Van der Velde et al. [25] used the Buller study population to compare the Wells DVT rule [30] to the Oudega rule (Table 1) [9]. A VTE event occurred during the 3-month follow-up in seven patients with a low score $(\leq 3)$, and a negative POCT D-dimer finding was reported when patients were assessed using both the Wells rule (7 of $447,1.6 \%$; 95\% CI $0.7-3.3 \%$ ) and the Oudega rule (7 of $495,1.4 \% ; 95 \%$ CI $0.6-3.0 \%)$. More patients would have been referred for further testing using the Wells rule (581 patients, 55\%) compared with the Oudega rule (533 patients, 51\%; McNemar test, $P<0.001)$. Van der Velde et al. reported that the POCT D-dimer test alone had a sensitivity of $86 \%$ and a specificity of $61 \%$, which contributed to good clinical efficiency [25]. In these studies, the POCT D-dimer assay was reported to be easy to perform in the GP's office or at the patient's home, providing an instant and easyto-interpret outcome.

Janssen et al. employed the Buller study population to determine whether the performance of the decision rule could be improved by: (i) reducing the proportion of false negatives (missed diagnoses [safety]); or (ii) reducing the proportion of patients referred (efficiency) (Table 1). Inclusion of further variables such as 


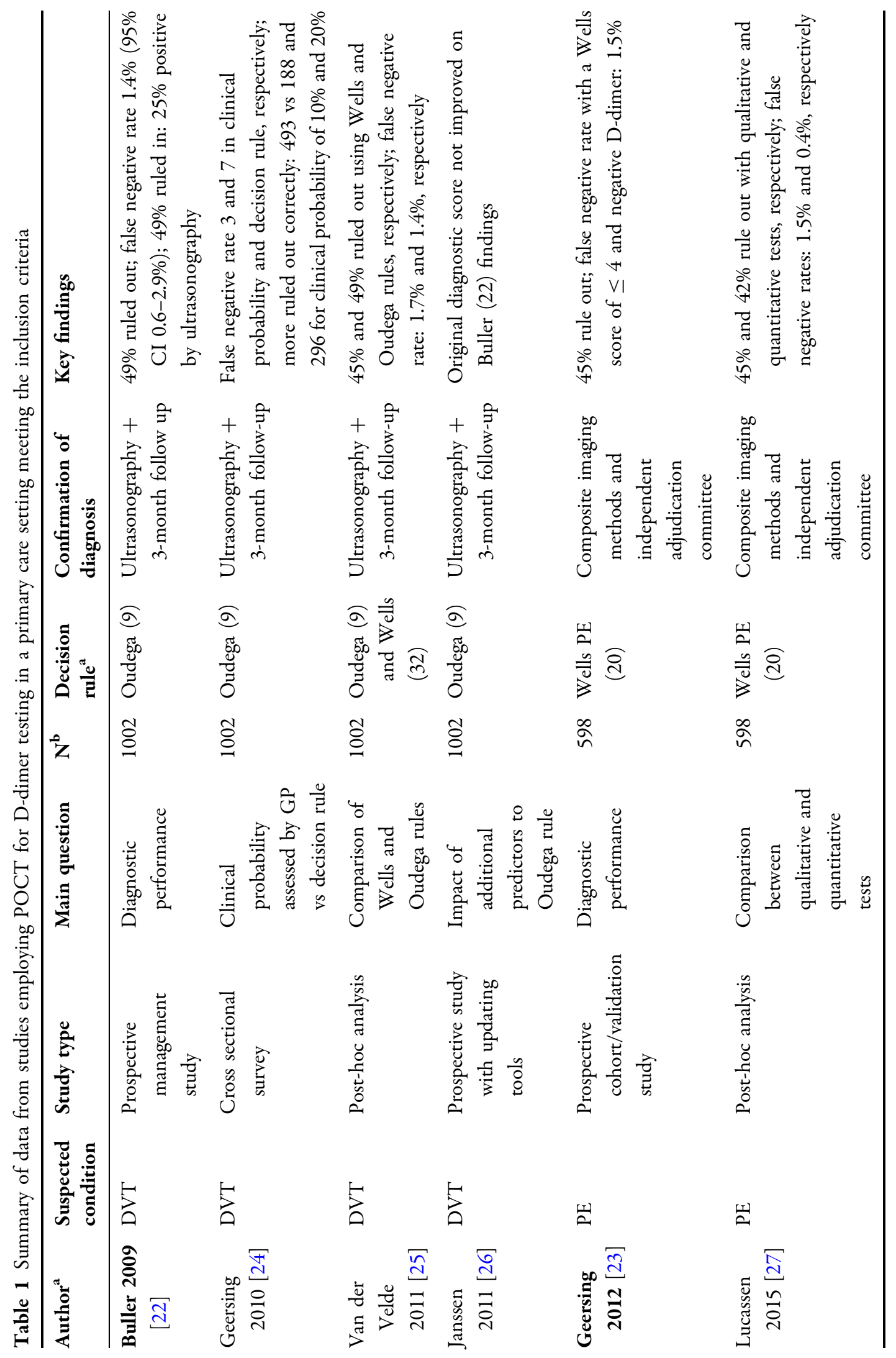




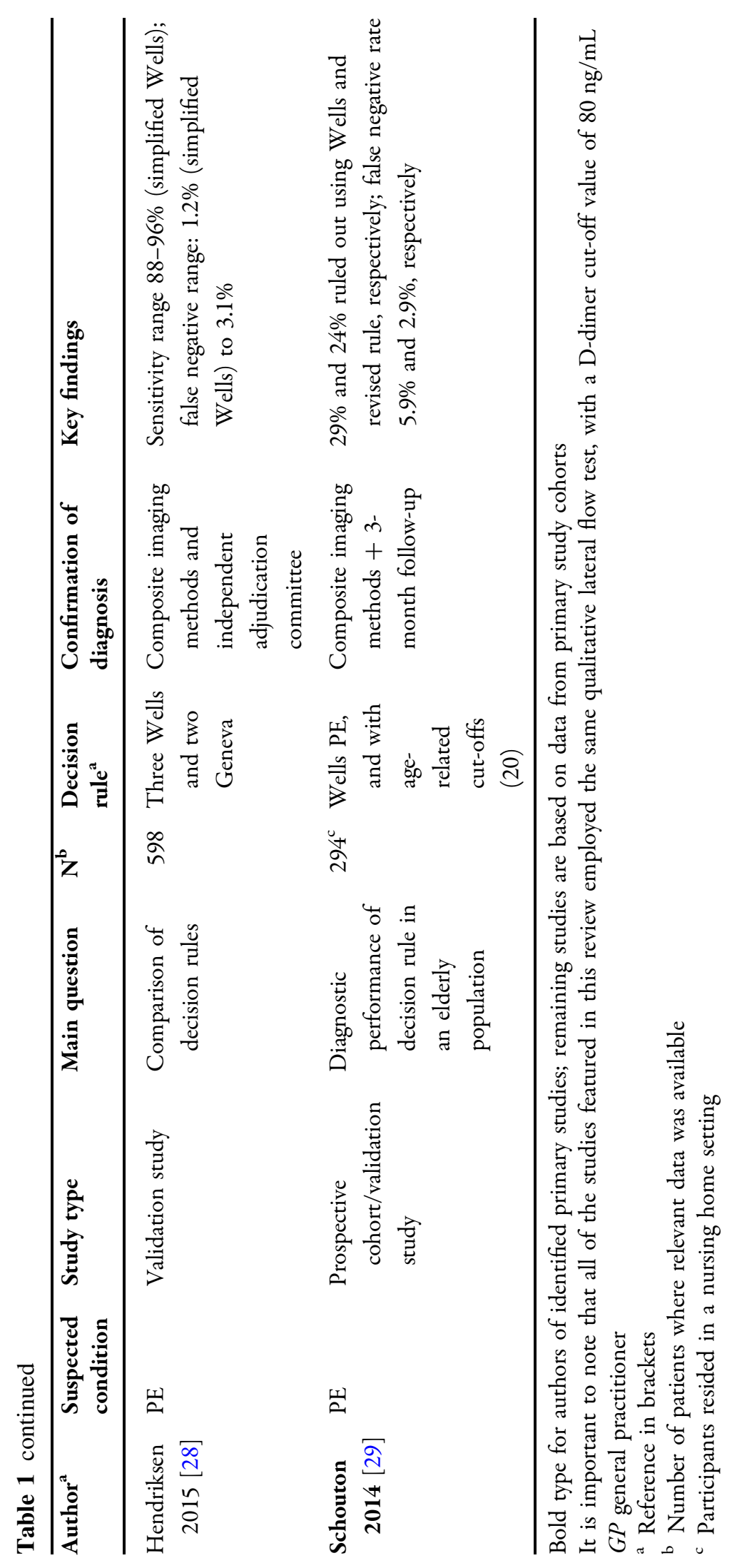


'history of DVT' and 'prolonged travelling' significantly increased the predictive value $(P=0.014$ and 0.023 , respectively), but did not improve safety and efficiency. The results indicated that at equal safety $(1.4 \%$ missed diagnoses among the non-referred patients), the efficiency was lower $(43.5 \%$; 95\% CI $40.4-46.6 \%$ vs $49.4 \%$; $95 \%$ CI $46.3-52.5 \%)$. The study suggested that the original diagnostic score can be used to safely exclude clinically suspected DVT in primary care [26].

\section{Clinical Effectiveness of POCT D-Dimer in PE}

Geersing et al. [23] recruited a cohort of 598 adults, with the participation of over 300 GPs, to validate the use of a simplified Wells clinical decision rule [19], combined with qualitative POCT for D-dimer, to safely exclude PE in primary care. All patients, irrespective of the results obtained in primary care, were referred to secondary care for reference testing, which included a range of imaging investigations and clinical probability assessment (with or without a POCT D-dimer test). The participants were also followed up for 3 months. The failure rate (false negative) of the Wells rule (with a threshold score of $\leq 4$ combined with a negative POCT D-dimer result) was 1.5\% (95\% CI 0.4-3.0\%). Furthermore, $45.5 \%$ of 598 patients with suspected PE in primary care were classified as low risk using these criteria. The authors highlighted that these results were in accordance with studies performed in secondary care [31]. The D-dimer results were not interpretable in $6.5 \%$ of the cohort; although POCT D-dimer testing could easily be conducted in a primary care setting, it was reported that interpretation of a result using the qualitative POCT assay was sometimes challenging.

Using the same study cohort, Hendriksen et al. [32] compared the performance of a clinical probability score (on a visual analogue scale of $0-100 \%)$ for PE with the use of a decision rule, including a POCT D-dimer result. They calculated a Wells score for each patient, ranging from 0 to 12.5 , with higher scores indicating a greater probability of PE. The results revealed that the Wells rule not only improved diagnostic performance, but also enabled PE to be ruled out in a higher proportion of patients ( $45 \%$ vs $25 \%$ ), offering greater efficiency and reducing the burden on local healthcare services (Table 1).

Lucassen et al. [27] undertook further analysis of the data collected in the Geersing study [23], comparing the performance of qualitative POCT and laboratory-based quantitative tests. The sensitivity and specificity of the Wells rule combined with POCT for D-dimer were 94.5\% and $51.0 \%$, compared to $98.6 \%$ and $47.2 \%$, respectively, for the laboratory-based quantitative test. They concluded that both tests were acceptable, together with the Wells score, to rule out a diagnosis of PE. Hendriksen et al. [28] used the same Geersing study data [23], together with a systematic review, to validate five decision models for ruling out PE in primary care (three versions of the Wells score and two of the Geneva score; Table 1). They suggested that GPs use the simple version of the Wells score combined with POCT for D-dimer and concluded that PE can be excluded in about four in every 10 patients with suspected PE, with an acceptably low failure rate below $2 \%$.

\section{Age-Related Cut-Off for D-Dimer in VTE}

Caution has been expressed regarding the generalisability of diagnostic strategies, including D-dimer assessment, as D-dimer levels are typically increased in the elderly population [33]. The use of an age-related cut-off for D-dimer has been advocated for the hospital setting for the benefit of "increasing the proportion of older patients in whom PE could be safely excluded", reducing the burden on these patients [34]. Schouten et al., in 2014, undertook a prospective cohort study of 294 older ( $\geq 60$ years), ambulatory adults, with $44 \%$ residing in a nursing home, to determine the accuracy of the Wells PE decision rule [29]. It was reported that $29 \%$ and $25 \%$ were ruled out using the original Wells rule [19] and the age-related revised rule, respectively. The false negative rates found were $5.9 \%$ (95\% CI $2.5-13 \%)$ and $2.9 \%$ (95\% CI $0.8-10 \%)$ for the Wells PE rule and age-related 
revised rule, respectively. They proposed that the higher failure rate with the Wells PE clinical decision rule may have been due to the higher prevalence of PE reported in the elderly population [29].

Schouten et al. [35] undertook a retrospective analysis of the elderly participants in the derivation and validation studies of a decision rule, including laboratory-based D-dimer assessment, developed for primary care patients in whom DVT was suspected [9, 11]. It was reported that using the age-dependent cut-off values for D-dimer results (age in years $\times 10 \mu \mathrm{g} /$ $\mathrm{L}$ for patients $>50$ years), they could rule out DVT in $47.8 \%$ (95\% CI $43.9-51.7 \%)$ of the patients, an increase of $5.7 \%$ compared to the use of the conventional threshold value, yet the false negative rate had also increased $(0.5 \%$ vs $0.3 \%$, respectively). This study concluded that by increasing the threshold, fewer patients would be referred (saving referrals); however, a higher percentage of DVT cases would be missed [35].

\section{Cost-Effectiveness of POCT for D-Dimer in DVT}

Ten Cate-Hoek et al. assessed incremental costeffectiveness using a Markov cost-effectiveness model, comparing the use of POCT for D-dimer combined with a clinical decision rule in primary care with a hospital-based approach to DVT diagnosis, using the data from studies published previously [22, 36]. The model revealed an average cost saving of $€ 138$ ( $\$ 185$ at the time of publication), with a quality-adjusted life years (QALY) loss of 0.002 ( $€ 3589$ vs $€ 3727$, per QALY) for the primary care approach. The study demonstrated that the diagnostic management strategy based on a clinical decision rule and a POCT D-dimer assay to exclude DVT in primary care was cost-effective when compared to hospital-based strategies [36]. This costeffectiveness analysis was updated by Hendriksen et al., in which the use of four different POCT devices, all considered to be user-friendly with regards to use in primary care, was investigated with similar findings reported [37].

\section{DISCUSSION}

To our knowledge, a total of four systematic reviews have to date investigated the use of D-dimer as part of clinical decision rules in the diagnosis of VTE [17, 38-40]; however, none of these reviews have focussed on the use of D-dimer as part of a clinical decision rule in the primary care setting. Two reviews have explored the use of POCT for D-dimer in the primary care setting, but were limited to patients presenting with suspected PE $[2,41]$. DVT is notably more common than PE in the primary care setting, but reviews on the value of POCT for D-dimer in all VTE are lacking. An understanding of the evidence base specifically for primary care is required as patients tend to present with a larger variety of less specific and less severe clinical presentations. Moreover, rapid decisions in the absence of easily accessible hospital diagnostics are required to prevent ongoing diagnostic uncertainty and poor patient outcomes. There is a lower prevalence of VTE and a lesser severity of the condition in the primary care setting, as the patient is likely to be seen at an earlier stage of the condition. It is, therefore, important to explore the diagnostic performance of POCT for D-dimer in a primary care setting to guide diagnostic decision-making.

The key findings from the two primary studies addressing the research question were that in $49 \%$ and $45 \%$ of the study populations with suspected DVT [22] and PE [23], respectively, the condition could be ruled out, with a false negative rate of $1.4 \%$ and $1.5 \%$, respectively (Table 1). Geersing et al. considered the fact that the false negative rate was lower than $2 \%$, and the CI crossed the pre-defined $2 \%$ limit as clinically significant. However, it should be remembered that both of the studies employed a qualitative D-dimer assay based on a cut-off of $80 \mathrm{ng} / \mathrm{mL}$. As no formal methods for power calculations of model validation studies exist, further discussion among clinicians is required regarding the proportion that can be considered acceptable as the upper limit [23]. Using POCT for D-dimer as part of a strategy to exclude DVT in primary care has also been found to be cost- 
effective compared to secondary care-based strategies [36].

\section{Strengths and Limitations of This Study}

The strength of this review is that the data reported are derived solely from studies in which patients have presented with suspected VTE in the primary care setting. While there are only two studies addressing the primary research questions, they represent contributions from a large number of GP practices. The review also focuses on the use of POCT for D-dimer, as this technology is distinct from laboratory D-dimer assessment methods and may have additional benefits within the primary care setting. There are certain limitations in the conduct of the studies that constitute the evidence base for this review. They include: (i) a heterogeneous population recruited in some of the studies, with potential contamination due to an age profile that may influence the baseline D-dimer value, such as elderly patients, or a previous history of VTE; (ii) variation in the choice of reference method (imaging) for confirming the diagnosis of VTE; (iii) those performing the reference method not necessarily blinded to the results of the index test, which may have resulted in bias; (iv) the reference test (imaging) not being undertaken on all patients; (v) use of a range of clinical decision rules; (vi) recruitment of patients with a previous history of either DVT or PE in some studies; (vii) a reliance on clinical follow-up to detect missed thrombotic disease; and (viii) the majority of the studies were performed in the Dutch healthcare system-this may impact on the generalisability to other healthcare systems. Furthermore, none of the studies included in this review adopted a randomised controlled trial approach with the majority employing a 'prospective management study' approach equating to a validation study. However, it may be argued that this approach was able to generate 'real-world evidence'.

\section{Barriers to the Adoption of POCT in Primary Care}

The introduction of POCT into primary care practice has been recognised as challenging in several surveys of GPs [42-45].

\section{Analytical Performance}

There have been numerous studies of analytical performance of POCT for D-dimer in addition to clinical studies. Oude Elferink et al. reported on a clinical evaluation of seven quantitative, laboratory D-dimer tests and one qualitative POC D-dimer test, which highlighted the poor harmonisation in calibration between assays, resulting in the need to use individualised decision threshold values [46]. This finding has also been observed in results from external quality assurance schemes for laboratory-based, quantitative tests [47], and there have been calls for suitable reference material [48]. These observations need to be considered when comparing data and harmonising local clinical guidelines for the use of POCT and laboratorybased services.

Lucassen et al. compared the diagnostic performance of qualitative (POCT in primary care) and quantitative (laboratory-based) assays, which indicated that the false negative rates were $1.5 \%$ and $0.4 \%$, respectively. The quantitative test appeared to be safer than the qualitative test; however, the difference was not statistically significant (Table 1) [27]. Geersing et al., in a diagnostic meta-analysis of four D-dimer assays evaluated in varying outpatient settings, also found that the sensitivity was better using a quantitative assay (0.96 [95\% CI 0.91-0.98] and 0.93 [95\% CI 0.88-0.97]) compared with qualitative POCT assays $(0.87$ [95\% CI 0.81-0.91] and 0.85 [95\% CI 0.78-0.90]) [49]. Geersing et al. analysed data of 577 consecutive primary care patients with suspected DVT in a primary care diagnostic centre; clinical decision rule was omitted while forming the diagnosis. The group compared the diagnostic performance of four quantitative and one qualitative POCT D-dimer assay against a reference comparator (CUS) [50]. The quantitative assays employed different sample types (two with 
citrated plasma, one with citrated blood, and the other with lithium-heparinised blood). The D-dimer cut-off values varied from 196 to $570 \mu \mathrm{g}$ fibrinogen equivalent units (FEU); laboratory workers performed all of the tests, albeit with no previous experience of POCT. The sensitivities reported with the quantitative tests varied between 94 and 99\%, with the specificities between 39 and $62 \%$. The qualitative POCT device demonstrated a sensitivity of $91 \%$ and a specificity of $64 \%$. This study also included a questionnaire on the user-friendliness of the devices, conducted with 20 nurses from a thrombosis service. The main issue raised related to the interpretation of the results-judged to be problematic in $25 \%$ of the responses regarding the qualitative POCT device, and $0-5 \%$ in the case of the quantitative assays.

Our findings are similar to those reported by the evidence review supporting the revised NICE guideline in patients with suspected DVT, with comparable high sensitivities between POCT and laboratory-based D-dimer tests, with the evidence considered to be of low quality [20]. Evidence supporting the revised NICE guidelines in patients with suspected PE suggests that POCT D-dimer devices offer lower sensitivity $\quad(88 \% \quad[0.84-0.91] \quad$ vs $93 \%$ [0.91-0.94]), but higher specificity (63\% [0.57-0.69] vs 48\% [0.43-0.53]) compared with laboratory-based tests [20]. Crucially for the study aim of this NICE review, these results were based on patient presentations in a range of clinical settings, as well as a range of POCT technologies, with varying performance characteristics [20]; thus, no conclusions relating to the use of POCT in primary care could specifically be drawn.

The majority of the studies of POCT for D-dimer in primary care utilised qualitative POCT systems [22, 23]. Recently, a comparison of the analytical performance of five quantitative POCT assays with a hospital reference test showed that four performed analytically well with a set of 238 plasma samples from patients clinically suspected of VTE in general practice. Most devices were considered easy to use in a primary care setting [51]. In addition to the potentially superior diagnostic performance offered by the quantitative POCT assays enabling closer harmonisation with the local laboratory D-dimer assay, such tests would allow the use of age-related cut-off values for the older population. The application of an ageadjusted D-dimer threshold is regarded as improving the management of patients with suspected VTE, especially with access to direct oral anticoagulants [52]. We would expect clinical performance of decision rules to be improved with the use of quantitative D-dimer tests, as well as age-adjusted cut-off points; this can only be proved with further studies.

\section{Implementation Plan}

Implementation of POCT is recognised as a challenge and has been evaluated in a POCTfacilitated diagnostic pathway for DVT in primary care involving 450 GPs, with the aid of educational outreach visits, financial reimbursements and periodical newsletters [53]. The researchers addressed 'acceptability', 'feasibility', 'fidelity' and 'sustainability'. The study showed an increase in the use of the pathway from $42 \%$ to an expected continuation of use of $91 \%$. Regarding clinical outcomes, $54 \%$ of patients were not referred to the hospital, missing six cases of DVT $(1.8 \% \quad[95 \%$ CI $0.7-3.9 \%]$ ), reflecting similar observations of other studies. However, it was also noted that during the implementation study, the pathway guidelines were found to have not been used correctly for $32 \%$ of patients. The researchers concluded that the study had demonstrated evidence of high acceptability, feasibility and expected sustainability. Interestingly, the implementation strategy described by Kingma et al. [53] included reimbursement, addressing one of the concerns expressed in the adoption of POCT in primary care $[42,44]$.

\section{Investment and Disinvestment}

A total of five case studies reported the redesigning of the local DVT pathway by transferring the diagnosis and treatment of non-complex DVT patients into primary care to avoid unnecessary hospital admissions; four of these five case studies included measurements using POCT D-dimer devices [21]. A consistent finding in the case studies was the reduction in 
the number of patients referred to the hospital for diagnosis or anticoagulation management, and greater patient satisfaction. There was also a significant reduction in cost to local healthcare services, despite the need to purchase diagnostic equipment. Of note, very limited quantitative data were provided in these studies [21].

Evidence from a cost-consequence model developed in support of NICE guidelines indicated that POCT for D-dimer results in a small, statistically significant increase (4 per 1000 people) in the number of false negative results and a large, statistically significant decrease (138 per 1000) in the number of false positive results. Excluding primary care costs, the overall POCT strategy was found to be less costly than laboratory testing $(-£ 1331$ [95\% credible interval, $-£ 10,777$ to $£ 8721]$ ). When primary care costs are included, the overall POCT strategy becomes significantly less costly $(-£ 20,166$ [95\% credible interval, $-£ 30,296$ to $-£ 9527]$ ) [20]. Clearly this data will need to be updated when studies employing a quantitative POCT tests have been completed.

Therefore, evidence of the potential cost-effectiveness of adopting POCT for D-dimer rests on demonstrating: (i) a reduction in the number of patients with suspected VTE requiring referral to hospital for further imaging investigations; and (ii) a reduction in the number of patients admitted to hospital with complications of VTE due to a failure to make a timely diagnosis.

\section{CONCLUSIONS}

The evidence, albeit limited in terms of the number of studies, indicates that POCT for D-dimer can be employed in the primary care setting to reliably guide diagnostic and management strategies for patients presenting with suspected VTE, reducing the time to diagnosis and treatment. A good D-dimer POCT device used to rule out a diagnosis could safely reduce DVT and PE referrals to hospital. Some of these cost savings can be used to invest in POCT technology and infrastructure. Evidence suggests that a quantitative POCT assay is the preferred technology choice; additionally, this will enable the use of age-related cut-off values. The choice of device, and its associated cut-off values, should be harmonised with the service provided by the laboratory serving the local hospital. Furthermore, the operators of the D-dimer testing system in primary care should participate in regular quality control and quality assurance programmes, and collaborate with POCT experts from an accredited laboratory.

\section{ACKNOWLEDGEMENTS}

The literature database search was undertaken by Sushmita Roy Nawathe and Maria Haughton (both of imc integrated medical communications, UK), and sponsored by LumiraDx.

Funding. This study was supported through an educational grant from LumiraDx, which also funded the Rapid Service fee.

Authorship. All named authors meet the International Committee of Medical Journal Editors (ICMJE) criteria for authorship for this article, take responsibility for the integrity of the work as a whole, and have given their approval for this version to be published.

Disclosures. Christopher P Price has undertaken consultancy work for LumiraDx. Matthew Fay: Westcliffe Health Innovation, of which MF is a partner, has received funding or support from: Abbott, AstraZeneca, Bayer, BoehringerIngelheim, Bristol Myers Squibb, LumiraDx, Medtronic, Novartis Oberoi Consulting, Pfizer, Roche, Sanofi-Aventis, Servier, Zenicor. Matthew Fay is an advisor to: Anticoagulation UK, Arrhythmia Alliance, Heart Valve Voice, National Stroke Association, Syncope Trust. MF is a member of the: PCCS Executive, BIHS Educational Programs Working Group, LumiraDx Advisory Committee, and is a trustee of Thrombosis UK and AF Association. Rogier M Hopstaken has no conflicts of interest to declare.

Compliance with Ethics Guidelines. This article is based on previously conducted studies and does not contain any new studies with 
human participants or animals performed by any of the authors.

Data Availability. Data sharing is not applicable to this article as no datasets were generated or analysed during the current study.

Open Access. This article is licensed under a Creative Commons Attribution-NonCommercial 4.0 International License, which permits any non-commercial use, sharing, adaptation, distribution and reproduction in any medium or format, as long as you give appropriate credit to the original author(s) and the source, provide a link to the Creative Commons licence, and indicate if changes were made. The images or other third party material in this article are included in the article's Creative Commons licence, unless indicated otherwise in a credit line to the material. If material is not included in the article's Creative Commons licence and your intended use is not permitted by statutory regulation or exceeds the permitted use, you will need to obtain permission directly from the copyright holder. To view a copy of this licence, visit http://creativecommons.org/licenses/by$\mathrm{nc} / 4.0 /$.

\section{REFERENCES}

1. Heit JA, Spencer FA, White RH. The epidemiology of venous thromboembolism. J Thromb Thrombolysis. 2016;41:3-14.

2. Reynen E, Severn M. Point-of-care d-dimer testing: a review of diagnostic accuracy, clinical utility, and safety. Ottawa: CADTH; 2017. (CADTH rapid response report: summary with critical appraisal). https://www.cadth.ca/sites/default/files/pdf/htis/ 2017/RC0940\%20POC\%20D-Dimer\%20Testing\% 20Final.pdf. Accessed 17 Sept 2020.

3. Weitz JI, Fredenburgh JC, Eikelboom JW. A test in context: D-dimer. J Am Coll Cardiol. 2017;70: 2411-20.

4. Walen S, Damoiseaux RA, Uil SM, van den Berg JW. Diagnostic delay of pulmonary embolism in primary and secondary care: a retrospective cohort study. Br J Gen Pract. 2016;66(647):e444-50.
5. Hendriksen JM, Koster-van Ree M, Morgenstern MJ, et al. Clinical characteristics associated with diagnostic delay of pulmonary embolism in primary care: a retrospective observational study. BMJ Open. 2017;7(3):e012789. https://doi.org/10.1136/ bmjopen-2016-012789.

6. Smith SB, Geske JB, Maguire JM, et al. Early anticoagulation is associated with reduced mortality for acute pulmonary embolism. Chest. 2010;137: 1382-90.

7. Grosse SD, Nelson RE, Nyarko KA, et al. The economic burden of incident venous thromboembolism in the United States: a review of estimated attributable healthcare costs. Throm Res. 2016;137: 3-10.

8. Wells PS, Ihaddadene R, Reilly A, Forgie MA. Diagnosis of venous thromboembolism: 20 years of progress. Ann Intern Med. 2018;168:131-40.

9. Oudega R, Moons KGM, Hoes AW. Ruling out deep venous thrombosis in primary care. A simple diagnostic algorithm including D-dimer testing. Thromb Haemost. 2005;94:200-5.

10. Oudega R, Hoes AW, Moons KGM. The Wells Rule does not adequately rule out deep venous thrombosis in primary care patients. Ann Intern Med. 2005;143:100-7.

11. Toll DB, Oudega R, Bulten RJ, et al. Excluding deep vein thrombosis safely in primary care. Validation study of a simple diagnostic rule. J Fam Pract. 2006;55:613-8.

12. Konstantinides SV, Meyer G, Becattini C, ESC Scientific Document Group, et al. 2019 ESC Guidelines for the diagnosis and management of acute pulmonary embolism developed in collaboration with the European Respiratory Society (ERS). Eur Heart J. 2020;41:543-603.

13. Lim W, Le Gal G, Bates SM, et al. American Society of Hematology 2018 guidelines for management of venous thromboembolism: diagnosis of venous thromboembolism. Blood Adv. 2018;2:3226-56.

14. American Academy of Family Practitioners. Diagnosis of venous thromboembolism-clinical practice guideline. https://www.aafp.org/patient-care/ clinical-recommendations/all/venousthromboembolism1.html. Accessed 17 Sept 2020.

15. de Jong J, Lucassen WA, Geersing GJ, et al. NHG guideline deep-vein thrombosis and pulmonary embolus. Ned Tijdschr Geneeskd. 2015;159:A8657.

16. Mooney C, Byrne M, Kapuya P, et al. Point of care testing in general haematology. $\mathrm{Br} \mathrm{J}$ Haematol. 2019; 187:296-306. 
17. National Institute for Health and Care Excellence. Venous thromboembolic diseases: diagnosis, management and thrombophilia testing. NICE guideline [NG158]. https://www.nice.org.uk/guidance/ ng158. Accessed 17 Sept 2020.

18. Wells PS, Anderson DR, Rodger M, et al. Evaluation of D-dimer in the diagnosis of suspected deep-vein thrombosis. N Engl J Med. 2003;349:1227-35.

19. Wells PS, Anderson DR, Rodger M, et al. Derivation of a simple clinical model to categorize patients' probability of pulmonary embolism: increasing the model's utility with the SimpliRED D-dimer. Thromb Haemost. 2000;83:416-20.

20. National Institute for Health and Care Excellence. Venous thromboembolic diseases: diagnosis, management and thrombophilia testing $[\mathrm{A}]$ Evidence reviews for D-dimer testing in the diagnosis of deep vein thrombosis and pulmonary embolism. https:// www.nice.org.uk/guidance/ng158/evidence/addimer-testing-in-the-diagnosis-of-deep-veinthrombosis-and-pulmonary-embolism-pdf8710588334. Accessed 17 Sept 2020.

21. All-Party Parliamentary Thrombosis Group. DVT diagnosis and treatment in primary care. http:// apptg.org.uk/wp-content/uploads/2016/12/NHSInnovation-Showcase.pdf. Accessed 17 Sept 2020.

22. Buller HR, Cate-Hoek AJT, Hoes AW, AMUSE (Amsterdam Maastricht Utrecht Study on thromboEmbolism) Investigators, et al. Safely ruling out deep venous thrombosis in primary care. Ann Intern Med. 2009;150:229-35.

23. Geersing G-J, Erkens PMG, Lucassen WAM, et al. Safe exclusion of pulmonary embolism using the Wells rule and qualitative D-dimer testing in primary care: prospective cohort study. BMJ. 2012;345:e6564. https://doi.org/10.1136/bmj. e6564.

24. Geersing G-J, Janssen KJ, Oudega R, on behalf of the AMUSE Study Group, et al. Diagnostic classification in patients with suspected deep venous thrombosis: physicians' judgement or a decision rule? Brit J Gen Pract. 2010;60:742-8.

25. van der Velde EF, Toll DB, Cate-Hoek AJT, et al. Comparing the diagnostic performance of 2 clinical decision rules to rule out deep vein thrombosis in primary care patients. Ann Fam Med. 2011;9:31-6.

26. Janssen KJM, van der Velde EF, ten Cate AJ, et al. Optimisation of the diagnostic strategy for suspected deep-vein thrombosis in primary care. Thromb Haemost. 2011;105:154-60.

27. Lucassen WA, Erkens PM, Geersing GJ, et al. Qualitative point-of-care D-dimer testing compared with quantitative D-dimer testing in excluding pulmonary embolism in primary care. J Thromb Haemost. 2015;13:1004-9.

28. Hendriksen JM, Geersing GJ, Lucassen WA, et al. Diagnostic prediction models for suspected pulmonary embolism: systematic review and independent external validation in primary care. BMJ. 2015;8(351):h4438. https://doi.org/10.1136/bmj. h4438.

29. Schouten HJ, Geersing G-J, Oudega R, et al. Accuracy of the Wells clinical prediction rule for pulmonary embolism in older ambulatory adults. J Am Geriatr Soc. 2014;62:2136-41.

30. Wells PS, Owen C, Doucette S, et al. Does this patient have deep vein thrombosis? JAMA. 2006;295:199-207.

31. Lucassen W, Geersing GJ, Erkens PM, et al. Clinical decision rules for excluding pulmonary embolism: a meta-analysis. Ann Intern Med. 2011;155:448-60.

32. Hendriksen JM, Lucassen WA, Erkens PM, et al. Ruling out pulmonary embolism in primary care: comparison of the diagnostic performance of "Gestalt" and the Wells rule. Ann Fam Med. 2016;14:227-34.

33. Schouten HJ, Koek HL, Moons KG, et al. Need for tailored strategies to diagnose venous thromboembolism in older primary care patients. Extension of a keynote presentation at the 2012 Wonca Europe conference. Eur J Gen Pract. 2013;2013(19): $123-7$.

34. Douma RA, le Gal G, Söhne M, et al. Potential of an age adjusted D-dimer cut-off value to improve the exclusion of pulmonary embolism in older patients: a retrospective analysis of three large cohorts. BMJ. 2010;340:c1475. https://doi.org/10.1136/bmj. c1475.

35. Schouten HJ, Koek HL, Oudega R, et al. Validation of two age dependent D-dimer cut-off values for exclusion of deep vein thrombosis in suspected elderly patients in primary care: retrospective, cross sectional, diagnostic analysis. BMJ. 2012;344: e2985. https://doi.org/10.1136/bmj.e2985.

36. Ten Cate-Hoek AJ, Toll DB, Büller HR, et al. Costeffectiveness of ruling out deep venous thrombosis in primary care versus care as usual. J Thromb Haemost. 2009;7:2042-9.

37. Hendriksen JM, Geersing GJ, van Voorthuizen SC, et al. The cost-effectiveness of point-of-care D-dimer tests compared with a laboratory test to rule out deep venous thrombosis in primary care. Expert Rev Mol Diagn. 2015;15:125-36. 
38. Stein PD, Hull RD, Patel KC, et al. D-dimer for the exclusion of acute venous thrombosis and pulmonary embolism: a systematic review. Ann Intern Med. 2004;140:589-602.

39. Di Nisio M, Squizzato A, Rutjes AWS, Büller HR, Zwinderman AH, Bossuyt PMM. Diagnostic accuracy of D-dimer test for exclusion of venous thromboembolism: a systematic review. J Thromb Haemost. 2007;5:296-304.

40. Crawford F, Andras A, Welch K, Sheares K, Chappell D. D-dimer test for excluding the diagnosis of pulmonary embolism. Cochrane Database Syst Rev. 2016;2016:CD010864.

41. Schols AMR, Stakenborg JPG, Dinant GJ, Willemsen RTA, Cals JWL. Point-of-care testing in primary care patients with acute cardiopulmonary symptoms: a systematic review. Fam Pract. 2018;35:4-12.

42. Jones C, Howick J, Roberts NW, et al. Primary care clinicians' attitudes towards point-of-care blood testing: a systematic review of qualitative studies. BMC Fam Pract. 2013;14:117.

43. Turner PJ, Van den Bruel A, Jones C, et al. Point-ofcare testing in UK primary care: a survey to establish clinical needs. Fam Pract. 2016;33:388-94.

44. Sohn AJ, Hickner JM, Alem F. Use of point-of-care tests (POCTs) by US primary care physicians. J Am Board Fam Med. 2016;29:371-6.

45. Kip MMA, Hummel JM, Eppink EB, et al. Understanding the adoption and use of point-of-care tests in Dutch general practices using multi-criteria decision analysis. BMC Fam Pract. 2019;20:8. https://doi.org/10.1186/s12875-018-0893-4.
46. Oude Elferink RF, Loot AE, Van De Klashorst CG, et al. Clinical evaluation of eight different D-dimer tests for the exclusion of deep venous thrombosis in primary care patients. Scand J Clin Lab Invest. 2015;75:230-8.

47. Tripodi A, Chantarangkul V. Performance of quantitative D-dimer methods: results of the Italian external quality assessment scheme. J Thromb Haemost. 2007;5:184-5.

48. Longstaff C, Adcock D, Olson JD, et al. Harmonisation of D-dimer-a call for action. Thromb Res. 2016;137:219-20.

49. Geersing GJ, Janssen KJ, Oudega R, et al. Excluding venous thromboembolism using point of care D-dimer tests in outpatients: a diagnostic metaanalysis. BMJ. 2009;339:b2990. https://doi.org/10. 1136/bmj.b2990.

50. Geersing GJ, Toll DB, Janssen KJ, et al. Diagnostic accuracy and user-friendliness of 5 point-of-care D-dimer tests for the exclusion of deep vein thrombosis. Clin Chem. 2010;56:1758-66.

51. Heerink J, Gemen E, Oudega R, et al. Analytical performance and user-friendliness of five novel point-of-care D-dimer assays. Scand J Clin Lab Invest. 2020;80:433-40. https://doi.org/10.1080/ 00365513.2020 .1768586 .

52. Tritschler T, Kraaijpoel N, Le Gal G, Wells PS. Venous thromboembolism: advances in diagnosis and treatment. JAMA. 2018;320:1583-94.

53. Kingma AEC, van Stel HF, Oudega R, et al. Multifaceted implementation strategy to increase use of a clinical guideline for the diagnosis of deep venous thrombosis in primary care. Fam Pract. 2017;34: $446-51$. 